\section{Therapieoptionen des subakuten lumbosakralen Reizsyndroms}

\author{
Epidurale Steroide, Etanercept oder physiologische Kochsalzlösung? Ist bei \\ Patienten mit subakuter Ischialgie die Injektion von Steroiden oder eines \\ Hemmers der Freisetzung von Tumornekrosefaktor-alpha (TNF alpha) in der \\ Schmerztherapie wirksam?
}

$C$ hrom hronische Rückenschmerzen und lumbosakrale radikuläre Schmerzen sind außerordentlich häufig und gehen mit hohen Kosten für das Gesundheitssystem einher. Von Seiten der Orthopäden und Schmerztherapeuten wird nach wie vor immer wieder propagiert, Patienten mit derartigen Beschwerden mithilfe lokaler Injektionen von Steroiden und/oder Lokalanästhetika zu behandeln - obwohl fast alle bisher durchgeführten randomisierten Studien keine Langzeitwirkung dieses Behandlungsansatzes gezeigt haben. Eine Reihe von Tierexperimenten legt nahe, dass es durch Bandscheibenvorfälle und Wurzelkompressionen und die dadurch ausgelöste Entzündungsreaktion zur Freisetzung von TNF alpha kommt. In Tierexperimenten war der TNF-alphaHemmer Etanercept entzündungshemmend wirksam.

Die multizentrische randomisierte und placebokontrollierte Studie wurde in den Jahren von 2008 bis 2011 durchgeführt. Daran teilgenommen hatten insgesamt 84 Patienten mit subakuten Rückenschmerzen und radikulären Schmerzen, die weniger als sechs Monate angehalten hatten.

Im Abstand von zwei Wochen wurde den Patienten jeweils eine Injektion mit $60 \mathrm{mg}$ Methylprednisolon, $4 \mathrm{mg}$ Etanercept oder physiologischer Kochsalzlösung verabreicht. Die Injektionsmenge war mit $2 \mathrm{ml}$ jeweils identisch. Alle Patienten erhielten 0,5 ml Bupivacain. Bei den Patienten, bei denen zwei Nervenwurzeln betroffen waren, wurde die Menge des zu injizierenden Medikaments halbiert und in zwei verschiedene Höhen injiziert.

Der primäre Endpunkt war die Schmerzintensität auf einer numerischen Ratingskala, wobei der in das Bein ausstrahlende Schmerz getrennt vom lokalen Rückenschmerz evaluiert wurde.
Zusätzlich wurde der Oswestry-Disability-Index (ODI) erhoben. Die primären Endpunkte wurden einen Monat nach der zweiten Injektion getestet sowie neuerlich nach drei und sechs Monaten.

Physiologische Kochsalzlösung erhielten 30 Patienten, epidurale Steroide 28 Patienten und epidurales Etanercept 26 Studienteilnehmer. Das mittlere Alter des Studienkollektivs betrug 42 Jahre. $70 \%$ der in die Studie eingeschlossenen Patienten waren Männer. Die Schmerzen hielten im Durchschnitt seit 2,5 bis drei Monaten an. Bei fast allen Patienten fand sich ein L5/S1-Syndrom. Etwa die Hälfte der Patienten erhielt eine Opioidtherapie, die auch während der Studie weiter fortgeführt wurde.

Vier Wochen nach der zweiten Injektion ergab sich für den Wert auf der numerischen Ratingskala für ausstrahlenden Schmerz in das Bein und lokalen Rückenschmerz kein statistisch signifikanter Unterschied zwischen den Therapiegruppen. Bezogen auf den ODIScore waren Steroide signifikant wirksamer als Etanercept, aber nicht signifikant effizienter als physiologische Kochsalzlösung. Nach drei und sechs Monaten ergaben sich ähnliche Ergebnisse. Auch konnten keine statistisch signifikanten Unterschiede in den sekundären Endpunkten festgestellt werden.

Fazit: Die epidurale Injektion von Steroiden bei radikulären lumbosakralen Schmerzsyndromen hat einen marginalen Kurzzeiteffekt, aber keine Langzeitwirkung. Der TNF-alpha-Hemmer Etanercept ist bei diesem Krankheitsbild nicht wirksam.

Cohen SP, White RL, Kurihara C et al. Epidural steroids, etanercept, or saline in subacute sciatica: a multicenter, randomized trial. Ann Intern Med 2012; 156: 551-9

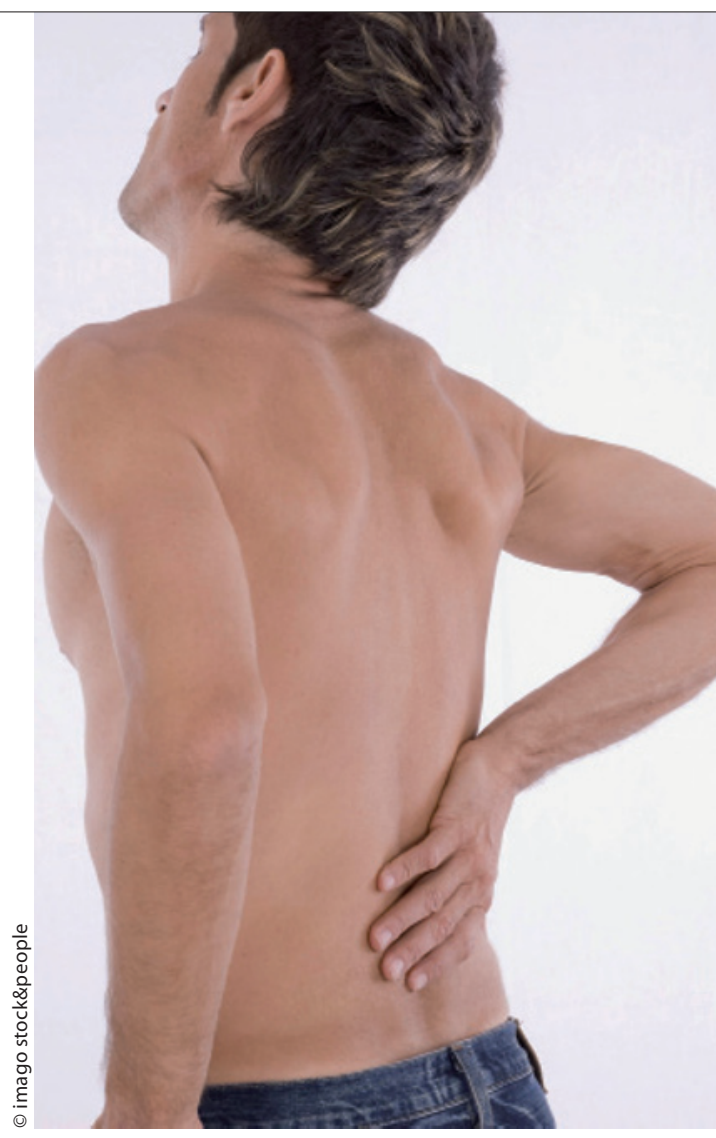

Chronische Rückenschmerzen - eine Krux auch für das Gesundheitssystem

Kommentar: Die hier vorliegende Studie weist zahlreiche methodische Probleme auf, wie beispielsweise kleine Patientengruppen und - bezogen auf den primären Endpunkt - vielleicht eine zu kurze Beobachtungszeit. Die Ergebnisse für die lokale Injektion von Steroiden replizieren die Resultate von vielen anderen randomisierten Studien, die ebenfalls keinen lang anhaltenden Behandlungserfolg fanden. Für weitere Studien ist es allerdings wichtig, das Etanercept ganz offenbar nicht wirkt und es daher wohl auch nicht sinnvoll ist, weitere Studien mit diesem innovativen Behandlungsansatz zu planen. 